\title{
Learning curve in rat dissection for experimental sciatic nerve repair
}

\section{Marin Andrei ${ }^{1}$, Marin Georgiana Gabriela ${ }^{2}$, Dobrete Nicoleta Amalia ${ }^{3}$, Enescu Dan Mircea ${ }^{4}$}

\author{
1 Plastic Surgery Department, "Bagdasar Arseni" Emergency \\ Hospital, Bucharest, ROMANIA \\ ${ }^{2}$ Cardiology Department, "CC Iliescu" Hospital, Bucharest, ROMANIA \\ 3 Haematology Department, "Fundeni" Hospital, Bucharest, ROMANIA \\ 4 Plastic Surgery Department, "Grigore Alexandrescu" Emergency \\ Paediatric Hospital, Bucharest, ROMANIA
}

\begin{abstract}
The baseline for any key research in nerve regeneration is an experimental model and the sciatic nerve in the rat model is the workhorse in this field. Although physically resistant to external traumas, a surgical intervention constitutes a major distress even for a rat. In the following presentation, we will analyse the learning curves for different stages in the rat sciatic nerve surgery as well as possible factors which influence these times.
\end{abstract}

\section{INTRODUCTION}

Every major breakthrough discovery starts from research. In surgery, it is immoral, unethical and sometimes even illegal for experiments which can be performed on animals to be done directly on humans. For this reason, animal experiments in the field of modern medicine are a necessity and they constitute the starting point for most of the innovative techniques in the surgical field. When it comes to nerve regeneration, the preferred choice is the sciatic nerve in the rat model.

The reason for this choice is that the sciatic nerve has a reasonable size $(1-3 \mathrm{~mm}$ in diameter), is fairly easily accessible (being located underneath the gluteus maximus, at the intersection between this muscle and the biceps femuri muscle, running its course between the knee joint and the ischial tuberosity) and because it divides towards the knee joint into its 3 main branches (common peroneal nerve, tibial nerve and sural nerve).

These 3 branches can be large enough for individual repair, but the sciatic nerve has proximal to this division sufficient length for different types of operations (grafts, nerve conducts), where an artificial gap can be created.

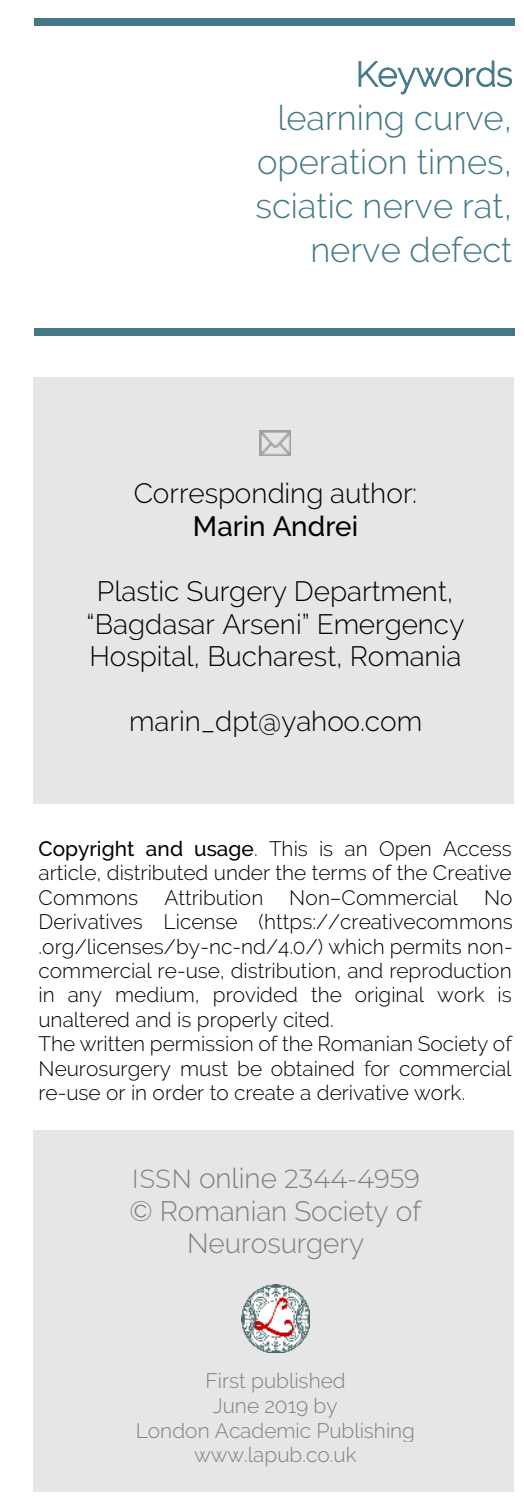




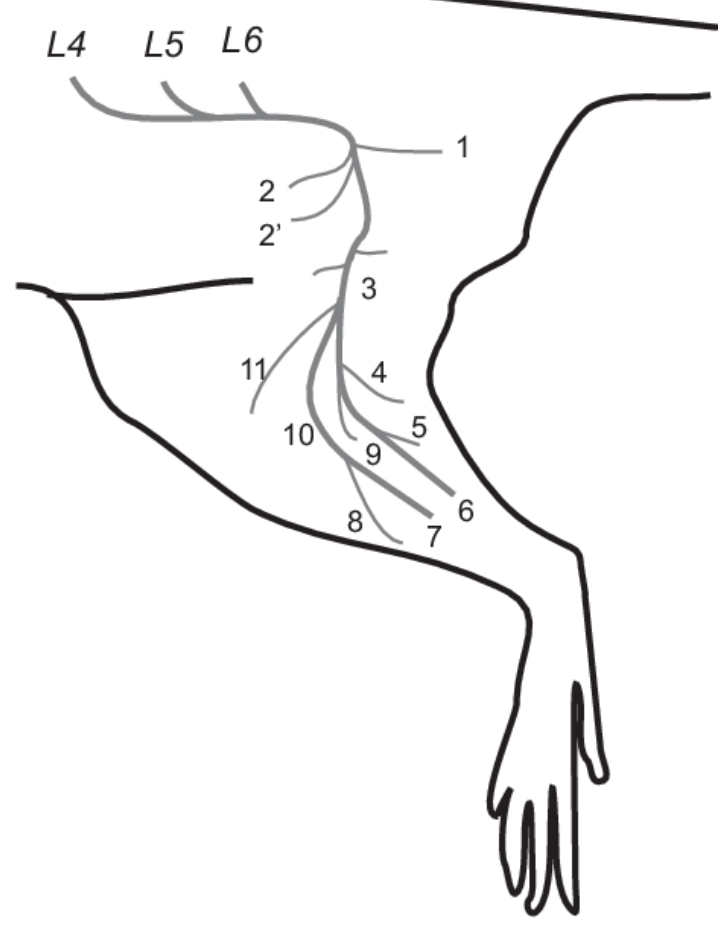

MATERIAL AND METHOD

For the experiment 42 Wistar male rats were used. All rats weighed between $240 \mathrm{~g}$ and $310 \mathrm{~g}$ and were 50-62 days of age at the time of surgery. All conditions regarding the safety and the well-being of the animals have been met. 2 rats were sacrificed to harvest vascular conducts and blood for PRP processing and the other 40 rats were divided into 4 batches of 10 . The learning curve presented in this article was designed for the first 2 batches (20 rats).

The operations were performed under general anaesthesia. The rat was positioned in supine position and an incision on the right posterior gluteal-thigh region was performed, followed by an incision of the biceps femuri muscle with sciatic nerve exposure. In the first batch, a distal section of the nerve was created, followed by nerve repair, proximal section of the sciatic with nerve anastomosis (by doing so, a controlled nerve graft of $0,5 \mathrm{~cm}$ in size was created in each rat, which was then sutured back into its original place). A 5th grade Sunderland lesion - neurotmesis was created at 2 different sites and then repaired. [3]

In the 2 nd batch, a $0,5 \mathrm{~cm}$ nerve defect was created and then solved using a vascular conduct. Batches 3 and 4 are part of a different study and involve adding PRP (platelet rich plasma) or human stem cells from the umbilical cord in the vascular conduct. [4],[5],[6].

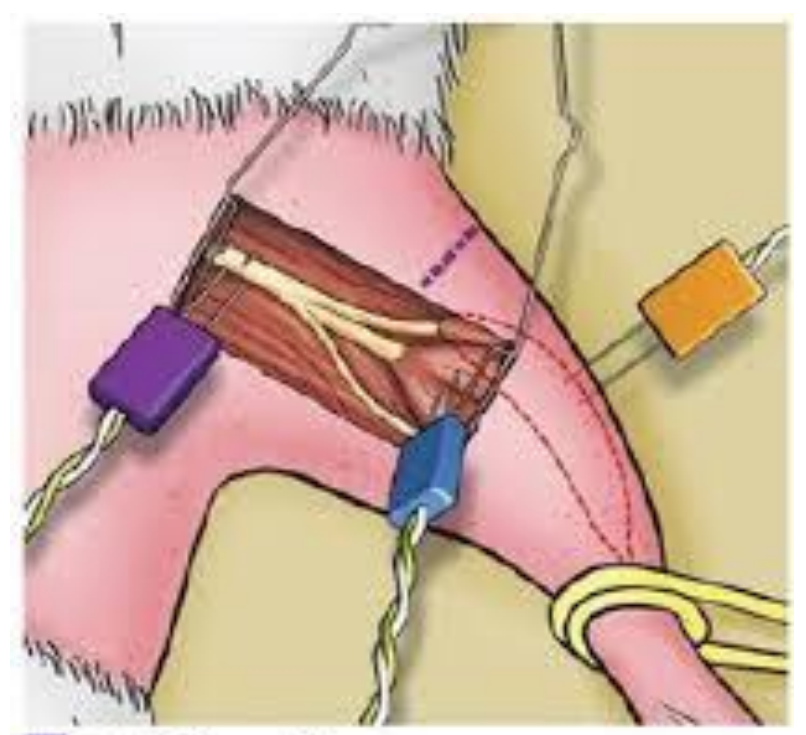

Sciatic Nerve Stimulus

\section{Gastrocnemius muscle recording}

Sciatic nerve and its divisions right leg of a rat: [1], [2]

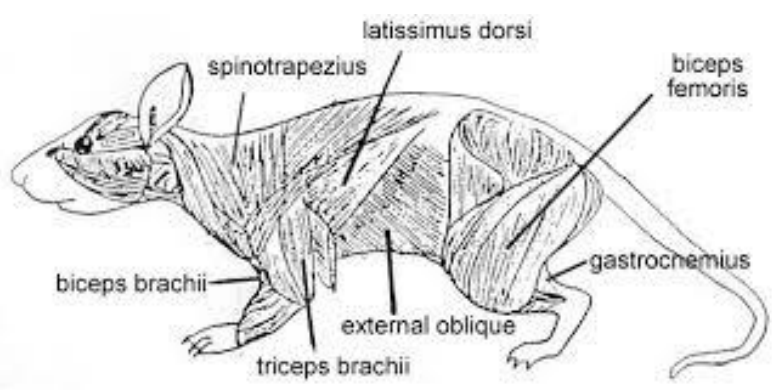

[7] Rat leg anatomy

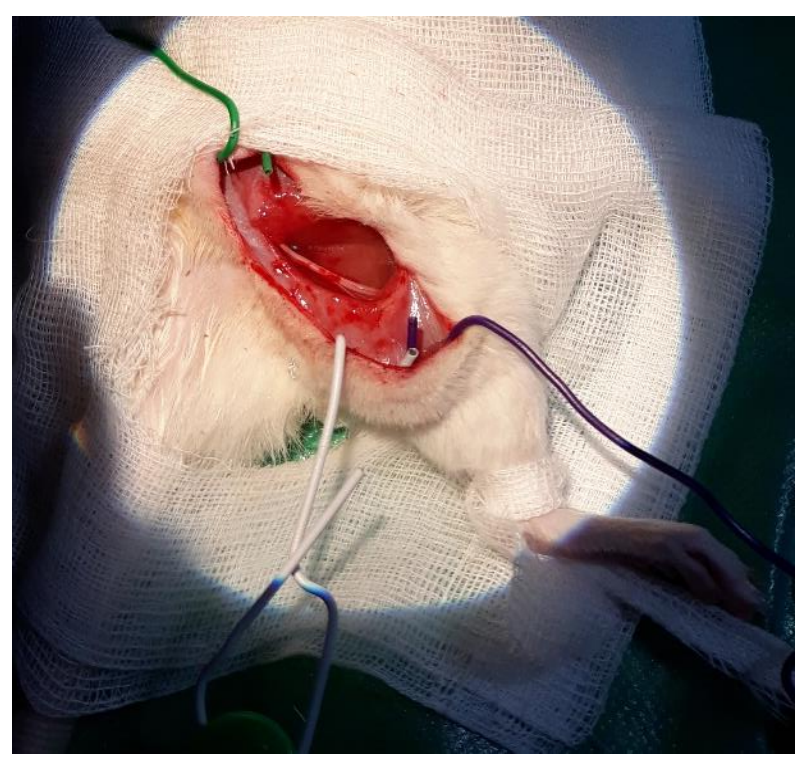




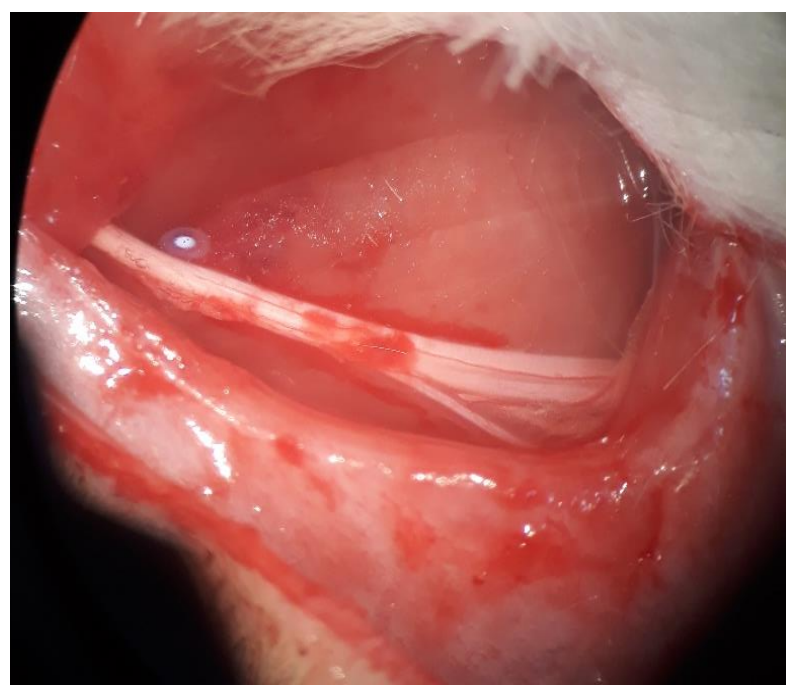

Sciatic nerve exposure

All interventions were performed in sterile conditions. An assistant was present for the duration of every operation to ensure the sterile conditions. All microsurgical anastomosis were performed using 10.0 Nylon suture under the microscope using a 10x magnification. The rats received 3 subcutaneous doses of meloxicam $0,04 \mathrm{ml}$ and 3 doses of enroxil $0,02 \mathrm{ml}(1$ immediately after the operation, and one for each of the next 2 days). Each rat was marked with circles on the tail and put in a separate cage.

Every procedure was recorded using a chronometer from the moment of the intraperitoneal injection till the skin suture. We observed the rat preparation times, the nerve exposure times and the anastomosis times.

The rat preparation time (T1) represents the time from the first doses of anaesthesia till the moment of incision and involves the time needed to shave the area which will be operated as well as time needed for setting the sterile field and the disinfection time. The nerve exposure time has 2 components: a macroscopic time for skin incision and muscle dissection till the nerve can be visualized (T2) and a microscopic time consisting in proper nerve dissection (T3). The $4 \mathrm{rd}$ registered time is the anastomosis time (T4)- the distal anastomosis followed by the proximal anastomosis. Once the nerve was completely repaired, muscle repair and skin suture finalized the intervention (T5). TT - total time - represents the sum of T1-T5.

The anaesthesia protocol consisted of using a mixture of ketamine $75 \mathrm{mg} / \mathrm{kg}$ and xylazine $10 \mathrm{mg} / \mathrm{kg}$ injected intraperitoneal. The anaesthesia normally worked in 5 minutes and one dose lasted between 20 and 50 minutes, when a new dose may have been required. Before the nerve dissection, as well as before nerve section, drops of lidocaine were supplementary used at the dissection site. The time for every anaesthesia dose administered was recorded and correlated to one of the 4 above mentioned times.

The rats in the 1st batch were operated in 3 separate days during the first week of the experiment ( 1 rat the first day, 4 rats the second day and 5 rats the 4th day of the week). The rats in the second batch were operated in 4 separate days over the second week of the experiment $(2$ rats the first day, 2 rats the 4 th day, 4 rats the 5th day and 2 rats the 6 th day).

Files for every operation were created in order to record all the data and for the assistant to remember the steps in the operations which they needed to fulfil (preparing the operating area, disinfection, adding lidocaine directly on the nerve once exposed, dropping saline solution in the eyes to prevent eye dryness and noting down all the previously mentioned times).

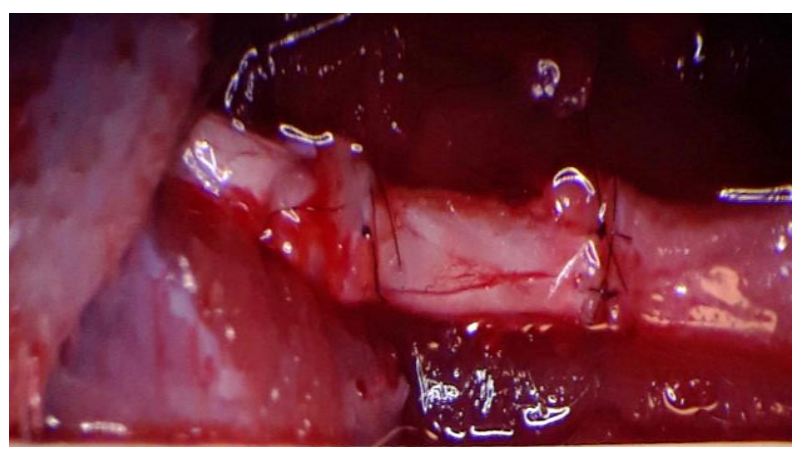

First batch - autograft

2nd batch - Vascular conduct

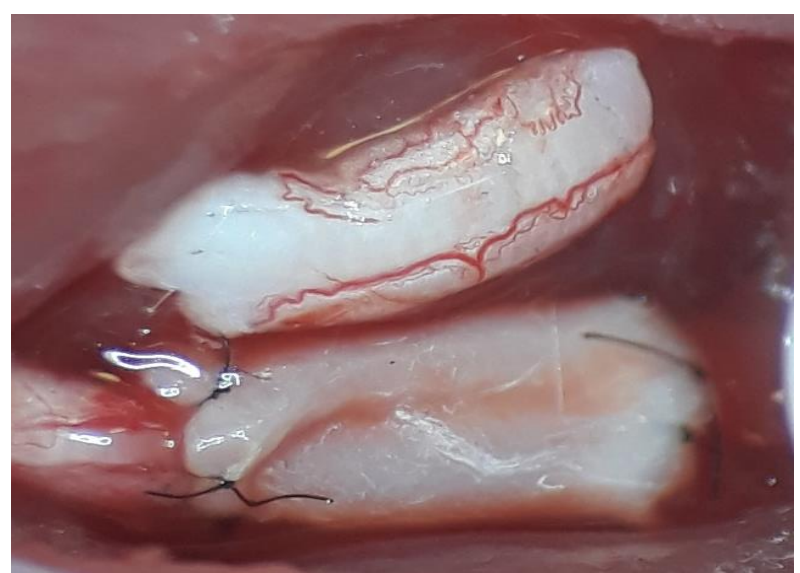




\section{RESULTS}

There is no statistical meaning between the 2 batches when referring to the median of age $(p=0.148)$ or weight $(p=0.264)$. Batch 1 ranged between 50-61 days of age at the time of the operation, while the weight ranged between 246$300 \mathrm{~g}$. In the second batch, the age varied between 50 and 53 days and the weight between 235-268g.

\begin{tabular}{|c|c|c|c|}
\hline Variable & $\begin{array}{l}\text { Batch } 1 \\
\text { Median } \\
(\mathrm{IQR})\end{array}$ & $\begin{array}{l}\text { Batch } 2 \\
\text { Median } \\
(\mathrm{IQR})\end{array}$ & $\begin{array}{r}\text { Total } \\
\text { Median } \\
\text { (IQR) } \\
\end{array}$ \\
\hline Age (days) & $52(4)$ & $51(1)$ & $55(2)$ \\
\hline $\begin{array}{l}\text { Weight } \\
\text { (gram) }\end{array}$ & $269(28)$ & 245 (18) & $\begin{array}{r}258.5 \\
(25)\end{array}$ \\
\hline T1 (min) & $43(17)$ & $23(9)$ & $35(21)$ \\
\hline T2 (min) & $5.5(2)$ & $7(8)$ & $6(5)$ \\
\hline T3 (min) & $9(4)$ & $8(6)$ & $8.5(5)$ \\
\hline T4 (min) & $37(9)$ & $29(16)$ & $35(11)$ \\
\hline$\pi T(\min )$ & $93(43)$ & $71.5(30)$ & $86(31)$ \\
\hline $\begin{array}{l}\text { No. } \\
\text { anesthesia }\end{array}$ & $2(1)$ & $2(1)$ & $2(1)$ \\
\hline $\begin{array}{l}\text { No. } \\
\text { sutures }\end{array}$ & $6.5(2)$ & $8(3)$ & $7(3)$ \\
\hline
\end{tabular}

There was a statistically significant difference between the preparation times ( $\mathrm{T} 1$ ) in the 2 batches $\mathrm{p}=0.005$ (the second batch having shorter preparation times compared to the first one). In the first batch, the T1 times improved from 136 minutes to 26 minutes, while the second batch had T1 times ranging from 37 to 19 minutes.

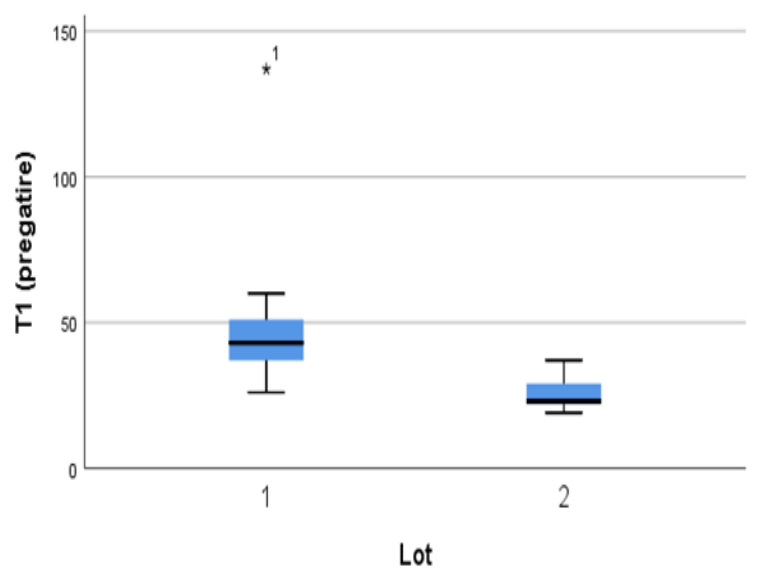

An overview of the variables recorded for the operations in the 2 batches shows:

1. number of doses of anaesthesia administered:
- batch 1 - median=2 (IQR:1), with minimum 2 and a maximum of 6 doses

- batch 2 - median=2 (IQR:1), with a minimum of 1 and a maximum of 3 doses

2. number of sutures for both proximal and distal anastomosis

- batch 1 - median = 6,5 (IQR:2), with a minimum of 4 and a maximum of 9 sutures

- batch 2 - median = 8 (IQR:3), with a minimum of 7 and a maximum of 12 sutures

After the statistical analysis (Pearson correlation), 2 positive correlation with statistical meaning were observed: on the one hand between the number of doses of anaesthesia administered and the total time of the operation for the 2 batches $(r=0.59, p=$ 0,007 ) - diagram $A$ and on the other hand between the number of doses of anaesthesia and the anastomosis time $\mathrm{T} 4(\mathrm{r}=0.65, \mathrm{p}=0.002)$-diagram $\mathrm{B}$.

The T4 times recorded for the 2 batches varied. For the first batch, there was a median of 37 minutes for the 2 anastomosis performed (with a maximum of 63 minutes and a minimum of 29 minutes), while in the second batch the median was at 29 minutes for 2 anastomoses (with a maximum of 60 minutes and a minimum of 13 minutes).

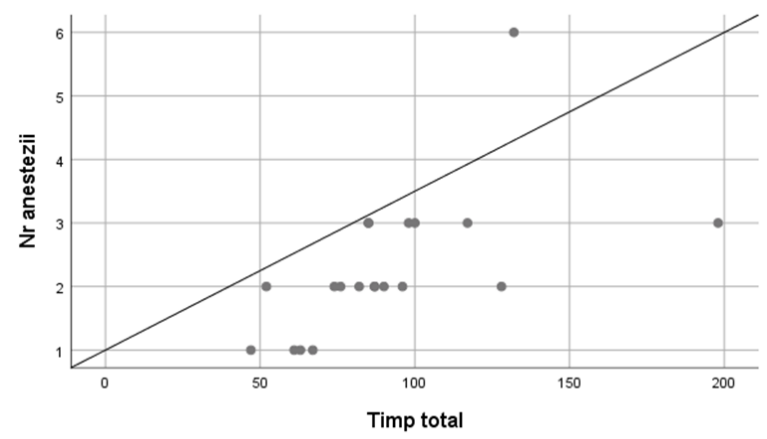

Diagram A

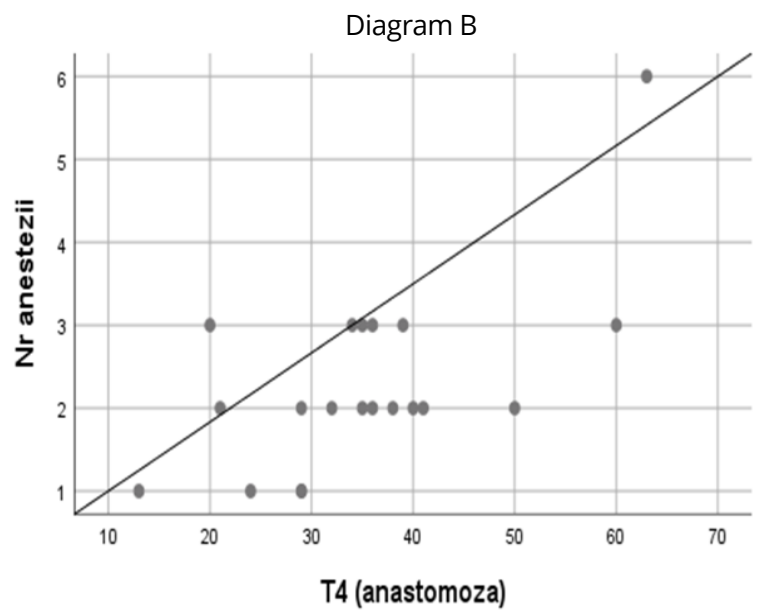




\begin{tabular}{|c|c|c|c|c|c|c|}
\hline \multirow[b]{2}{*}{ Correlation } & \multicolumn{2}{|l|}{ Batch 1} & \multicolumn{2}{|l|}{ Batch 2} & \multicolumn{2}{|c|}{ Total (batch1 + batch2) } \\
\hline & $\begin{array}{l}\text { Correlation } \\
\text { coef }(r)\end{array}$ & $\mathrm{p}$ & $\begin{array}{l}\text { Correlation } \\
\text { coef }(r)\end{array}$ & $\mathrm{p}$ & $\begin{array}{l}\text { Correlation } \\
\text { coef }(r)\end{array}$ & $\mathrm{p}$ \\
\hline $\begin{array}{l}\text { T4 - no. of } \\
\text { anaesthesia }\end{array}$ & 0.74 & 0.014 & 0.52 & 0.12 & 0.65 & 0.002 \\
\hline T4 - no. of sutures & 0.29 & 0.41 & 0.68 & 0.03 & 0.24 & 0.31 \\
\hline $\begin{array}{l}T T-\text { no. of } \\
\text { anaesthesia }\end{array}$ & 0.43 & 0.21 & 0.8 & 0.005 & 0.59 & 0.007 \\
\hline$T T-$ no. of suturi & 0.19 & 0.59 & 0.46 & 0.18 & 0.18 & 0.43 \\
\hline $\begin{array}{l}\text { No. of sutures - no. } \\
\text { of anaesthesia }\end{array}$ & 0.18 & 0.6 & 0.45 & 0.19 & 0.12 & 0.62 \\
\hline
\end{tabular}

No statistically significant correlations were established between the number of sutures and the anastomosis time T4 ( $r=0.24, p=0.31)$, number of doses of anaesthesia administered $(r=0,12, p=0.62)$ or total time $(r=0.18, p=0.43)$.

\section{DISCUSSIONS}

There are no scientific data regarding operation times in sciatic nerve surgery performed in the rat model. The correlation between the number of doses of anaesthesia administered and the total time of the operation is reasonable, taking into consideration that administrating a new dose of anaesthesia requires extra time; this is due to the fact that the rat was placed in supine position, while the anaesthesia was administered intraperitoneal (on the ventral side) by elevating the opposite inferior limb under sterile conditions.

The correlation between the number of doses of anaesthesia and the anastomosis time $\mathrm{T} 4 \mathrm{can}$ also be explained by the fact that the 2nd or 3rd dose of anaesthesia administered occurred mostly during $\mathrm{T} 4$, thus prolonging the anastomosis time.

Subjective factors may also influence the T2, T3 and T4 operation times (the overwork of the operator prolong the operating times) and resulted in fewer rats operated in a single day over the second week of the experiment.

The assistant who was partially in charge of shaving the rat after anaesthesia was able to considerably reduce the $\mathrm{T} 1$ time by improving his technique, as well as the operator who prepared simultaneously the sterile draping and suitable placed pins and retractors for the operation. This is how the rat preparation time was considerably reduced in the second batch.
The number of sutures might not have been correlated with the anastomosis time $\mathrm{T} 4$ because in the second batch this number was higher compared to the number of sutures performed in the first batch and while having similar or even faster times of execution. Therefore, when performing a higher number of sutures in similar times shows that the learning curve improved the timing of these anastomoses.

The only longer T4 time in the second batch of 60 minutes was in the case of the first rat, in which a new element was introduced - the bridging of the defect using the aortic conduct. All the other T4 times were under 40 minutes (compared to the first batch were 6/10 T4 times were under 40 minutes). This shows an improvement in the learning curve for the anastomosis, although no statistical difference was found. $(p=0,31)$.

\section{CONCLUSIONS}

The learning curve improved in the preparation time in the second batch compared to the first one, existing a statistical difference between the 2 batches. T4 - anastomosis times also improved in the second batch compared to the first one but this result wasn't statistically different.

Prolonged overall times were observed when a second or 3rd dose of anaesthesia was needed during the intervention. The anastomosis time and total operation time is directly proportional with the number of anaesthesia's performed for the anastomosis.

The learning curve also improved when referring to the anastomoses of the nerves, since a higher number of sutures was performed in similar or even shorter times. However, the number of sutures 
performed does not appear to influence the anastomosis time or the overall time in the given experiment.

\section{REFERENCES}

1. Prodanov, Dimiter \& Petkov, Dimiter. (2006). Morphometric analysis of the rat lower limb nerves: anatomical data for neural prosthesis design.

2. https://www.semanticscholar.org/paper/Tibial-andfibular-nerves-evaluation-using-in-rats.-NepomucenoPolitani/fcf3b6bbf5462ec73be2ee46987cc907abc03715/fi gure/5.

3. Andrei, M., Ioana, M., \& Mircea, E. (2019). Underlying histopathology of peripheral nerve injury and the classical nerve repair techniques. Romanian Neurosurgery, 33(1), 17-22. https://doi.org/10.33962/roneuro-2019-003.
4. Gärtner A, Pereira T, Armada-da-Silva P, et al. Effects of umbilical cord tissue mesenchymal stem cells (UCX®) on rat sciatic nerve regeneration after neurotmesis injuries. J Stem Cells Regen Med. 2014;10(1):14-26. Published 2014 Apr 30.

5. Sayad Fathi S, Zaminy A. Stem cell therapy for nerve injury. World J Stem Cells. 2017;9(9):144-151. doi:10.4252/wjsc. v9.i9.144.

6. Casal D, Mota-Silva E, Iria I, et al. Reconstruction of a 10$\mathrm{mm}$-long median nerve gap in an ischemic environment using autologous conduits with different patterns of blood supply: A comparative study in the rat. PLoS One. 2018;13(4):e0195692. Published 2018 Apr 16. doi:10.1371 /journal.pone.0195692.

7. https://www.biologycorner.com/worksheets/rat_external .html 